\title{
Correlation-Mediated Processes for Electron-Induced Switching between Néel States of Fe Antiferromagnetic Chains
}

\author{
Jean-Pierre Gauyacq \\ Institut des Sciences Moléculaires d'Orsay, ISMO, Unité mixte CNRS-Université Paris-Sud, \\ UMR 8214, Bâtiment 351, Université Paris-Sud, 91405 Orsay CEDEX, France \\ Simeón Moisés Yaro and Xavier Cartoixà \\ Departament de Enginyeria Electronica, Escola Tecnica Superior d'Enginyeria, \\ Universitat Autònoma de Barcelona, E-08193 Bellaterra, Spain \\ Nicolás Lorente \\ Centre d'Investigació en Nanociència i Nanotecnologia (CSIC-ICN), Campus de la UAB, E-08193 Bellaterra, Spain
}

(Received 7 November 2012; published 20 February 2013; corrected 7 March 2013)

\begin{abstract}
The controlled switching between two quasistable Néel states in adsorbed antiferromagnetic Fe chains has recently been achieved by Loth et al. [Science 335, 196 (2012)] using tunneling electrons from an STM tip. In order to rationalize their data, we evaluate the rate of tunneling electron-induced switching between the Néel states. Good agreement is found with the experiment, permitting us to identify three switching mechanisms: (i) low STM voltage direct electron-induced transitions, (ii) intermediate STM voltage switching via spin-wave-like excitation, and (iii) high STM voltage transitions mediated by domain-wall formation. Spin correlations in the antiferromagnetic chains are the switching driving force, leading to a marked chain-size dependence.
\end{abstract}

DOI: 10.1103/PhysRevLett.110.087201

PACS numbers: 75.10.Pq, 68.37.Ef, 75.50.Ee, 75.75.-c

The search for nanoscale electronic devices has prompted intense research in the field of nanomagnetism. The use of spin as the information conveying entity has stirred much excitement due to its extraordinary properties of information storage, speed, and low-energy consumption [1-3]. Miniaturization is quickly proceeding, reaching very small domain-wall devices [4], atomic-size devices [5], and the realm of molecular devices [2,6-9]. Among all these possibilities, antiferromagnetically (AFM) coupled devices have recently received a lot of attention. The AFM characteristics make these devices very well fitted for quantum computation since they naturally involved entangled states [10,11]. Moreover, the storage in AFM devices is particularly robust due to the lack of a total magnetic moment. However, this robustness has deterred their use because changing their magnetic state becomes difficult [12].

Recently, Loth and co-workers succeeded in controllably switching the spin states of AFM atomic chains [12]. Two quasistable Néel states, exhibiting alternating spin directions on the atoms along the chain, were evidenced in Fe chains adsorbed on a $\mathrm{CuN} / \mathrm{Cu}(100)$ surface. Loth and co-workers showed that the Néel states can be switched by tunneling electrons injected from a polarized scanning tunneling microscope (STM) tip into one of the atoms of the chain. This demonstrated the possibility of storing information on the atomic-scale antiferromagnet. Theoretical predictions show that writing and reading spin states entail fundamental problems associated with the quantum nature of the process [13]. Spin manipulation by tunneling electrons has been pictured as due to a spintorque mechanism where spin angular momentum from the electron is transferred into the atomic spin system [14-16]. However, due to the lack of magnetic moment in AFM systems, spin manipulation must follow a different mechanism. Unavoidably, spin manipulations and excitations are closely related [17]. Indeed, switching between Néel states has been experimentally associated with overcoming an activation energy [12]. However, in the case of degenerate Néel states, resonant transitions should be expected. Hence, the experimental data raise many questions regarding the possibility of resonant switching, the efficiency of activated switching, the nature of the involved excitations, and the physics at play in AFM spin torque. In summary, a complete view of the switching process is missing.

In this Letter, we reveal the switching mechanisms at play in the experiment of Ref. [12]. The mechanisms turn out to be rich and closely related with the excitation spectra of the AFM chain. Their understanding gives us a handle on the parameters controlling the switching process. Here, we show that the correlated spin nature of AFM quantal chains is at the origin of the transition between Néel states and, from this, we deduce the behavior of the switching rate with respect to applied bias and chain size. Hence, our theory shows that the ability of switching states is intrinsic to AFM-correlated atomic systems.

One $\mathrm{Fe}$ atom on $\mathrm{CuN} / \mathrm{Cu}(100)$ is characterized by an $S=2$ spin [18,19] with a large magnetic anisotropy [18]. 
The easy axis lies along the line of $\mathrm{N}$ atoms of the surface. Experimental [12,20] and theoretical [21] evidence shows that, in a first approximation, a chain of transition metal atoms on $\mathrm{CuN} / \mathrm{Cu}(100)$ is an ensemble of weakly interacting atoms such that the Fe chain can be described as a set of $S=2$ spins with a magnetic anisotropy and coupled by Heisenberg exchange coupling. This is partially due to the decoupling properties of the $\mathrm{CuN}$ layer $[14,16,20]$ and to the considerable distance between $\mathrm{Fe}$ atoms. Hence, the system can be described by the magnetic Hamiltonian

$$
\begin{aligned}
H_{0}= & \sum_{i=1}^{N-1} J \vec{S}_{i} \cdot \vec{S}_{i+1}+\sum_{i=1}^{N}\left[g \mu_{B} \vec{B} \cdot \vec{S}_{i}+D S_{i, z}^{2}\right. \\
& \left.+E\left(S_{i, x}^{2}-S_{i, y}^{2}\right)\right],
\end{aligned}
$$

where $\vec{S}_{i}$ is the spin of the atom $i(i=1, N)$ and $S_{i, u}$ is its projection on the $u$ axis. $D$ and $E$ are the longitudinal and transversal anisotropy coefficients $(D<0) . \vec{B}$ is a macroscopic magnetic field applied to the system along the $z$ axis and equal to $1 \mathrm{~T}$, as in the experiment [12]; tests revealed that the computed switching rate is very weakly dependent on the $B$ value.

Néel states are broken-symmetry solutions of Hamiltonian (1). Hence, Hamiltonian (1) cannot represent the Fe chains of Ref. [12] in the absence of an inhomogeneity that breaks the symmetry. In the present study, we introduced a phenomenological term to enforce the Néel magnetic structure. Hence, the magnetic Hamiltonian becomes

$$
H_{\mathrm{Mag}}=H_{0}+g \mu_{B} \vec{B}_{\text {inh }} \cdot \vec{S}_{1} .
$$

A small inhomogeneous field, $B_{\text {inh }}$, of $0.1 \mathrm{~T}$ acting on one of the atoms of the chain (here an end atom) is enough to split the ground states into two Néel-like states (denoted 1 and 2) that contain contributions from many spin configurations. This added perturbation is indeed small since the two Néel states are only $\sim 50 \mu \mathrm{eV}$ away from each other. The inhomogeneous term can be thought to be representative of various effects: small inhomogeneities of the surface or of the external $B$ field, induced by the polarized tip of the STM [22] or dephasing effects. Following Loth et al. [12], the parameters in Hamiltonian (1) were partially determined by fitting the experimental $\mathrm{Fe}_{2}$ excitation energies. Hence, similarly to the fitting of the Ising-model parameters in Ref. [12], we set $E=0$ and obtained $J=$ $1.6 \mathrm{meV}$ and $D=-1.34 \mathrm{meV}$ [23]. Here, we consider even-numbered chains, and the Hamiltonian (2) was diagonalized in a basis formed by direct products of the local spin states (spin configuration): $\Pi_{k}|M(k)\rangle$, where $|M(k)\rangle$ is an eigenstate of the projection on the $z$ axis of the local spin at atom $k, S_{k, z}$. For the longer chains, diagonalization methods specific to sparse matrices were used [24].

We describe the magnetic excitation during tunneling using the strong-coupling approach of Ref. [19] in which the electron strongly interacts with the atom it tunnels through. Let us consider the system schematized in Fig. 1(a): The STM tip is standing above the first atom of a chain in one of the two Néel states, and the injected electron is tunneling through it. Within the sudden approximation, the electron transmission amplitude operator is equal to [25]

$$
T_{\mathrm{Tip} \rightarrow \mathrm{Sub}}=\sum_{M_{T}}\left|S_{T}=5 / 2, M_{T}\right\rangle T_{\mathrm{Tip} \rightarrow \mathrm{Sub}}^{S_{T}}\left\langle S_{T}=5 / 2, M_{T}\right|,
$$

where $S_{T}$ is the spin of the compound system formed by the tunneling electron and the corresponding Fe atom. $T_{\text {Tip } \rightarrow \text { Sub }}^{S_{T}}$ is the electron transmission amplitude in the $S_{T}$ symmetry from tip to substrate. Here, we use $S_{T}=\frac{5}{2}$ revealed as the dominant tunneling channel by density functional theory studies in Ref. [19] for an isolated $\mathrm{Fe}$ atom on $\mathrm{CuN} / \mathrm{Cu}(100)$. The scattering amplitude between the chain magnetic states is then obtained as the matrix element of the amplitude (3) between initial $|i\rangle=\left|\sigma_{i}\right\rangle\left|\phi_{i}\right\rangle$ and final $|f\rangle=\left|\sigma_{f}\right\rangle\left|\phi_{f}\right\rangle$ states written as direct products of the scattering electron state of spin $\sigma$ and the chain state $|\phi\rangle$, the eigenstate of Hamiltonian (2). The probability, $P(i \rightarrow f)$, for a transition from the initial state $|i\rangle$ to the final state $|f\rangle$ associated to the tunneling of an electron from tip to substrate is proportional to $\left|T_{\mathrm{Tip} \rightarrow \mathrm{Sub}}^{S_{T}}\right|^{2}$ and to $\left|\sum_{M_{T}}\left\langle f \mid S_{T}=5 / 2, M_{T}\right\rangle\left\langle S_{T}=5 / 2, M_{T} \mid i\right\rangle\right|^{2}$. The $\left|T_{\text {Tip } \rightarrow \text { Sub }}^{S_{T}}\right|^{2}$ factor corresponds to a global tunneling probability, whereas the second factor yields the relative importance

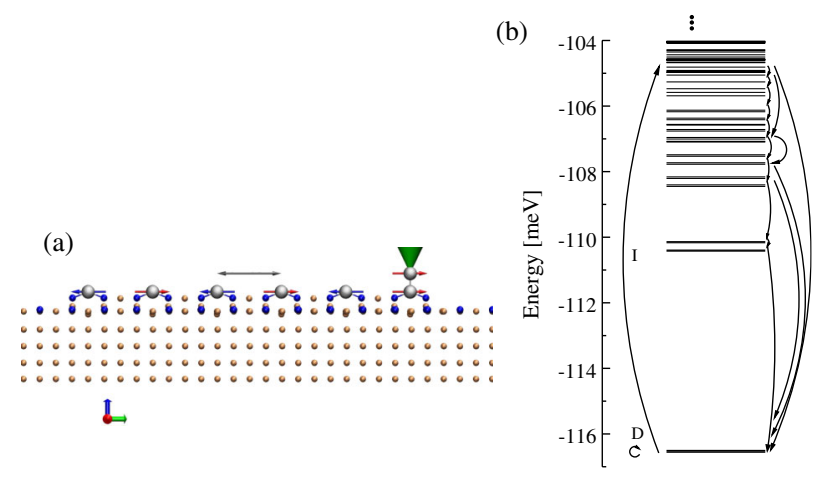

FIG. 1 (color online). (a) Setup used in the experiment of Ref. [12]. A few atoms of an Fe monoatomic chain on $\mathrm{CuN} / \mathrm{Cu}(100)$ are shown under an STM tip that injects spinpolarized electrons. The chain spins alternate along the chain axis to form a Néel state. (b) Efficient excitation and deexcitation schemes leading to the switching between Néel states. $D$ refers to the direct process between the quasidegenerate Néel states, and $I$ refers to the indirect one that involves excitation and deexcitation mechanisms. The up arrow on the left shows the excitation by a tunneling electron, whereas the down arrows on the right show the relaxation induced by substrate electrons. All possible deexcitation cascades are included in the numerical study, we schematically show a few deexcitation paths. 
of the inelastic channels in the tunneling process. The $(i \rightarrow f)$ probability is very large if there is a strong overlap between the intermediate state of $S_{T}$ tunneling symmetry and both the initial and final states.

The electron can only induce a change of one unit in the spin projection of the atom it tunnels through. As a consequence, in a zero-order view of the Néel states, described as chains of atoms with alternating spins, electron-induced switching between pure Néel states does not exist. However, AFM chains described with Heisenberg couplings are strongly correlated [26,27]; i.e., the two Néellike states, eigenstates of Hamiltonian (2), contain small components over a very large number of different configurations of atomic spins. Direct, quasiresonant electroninduced transitions between Néel-like states are then possible. These quantal transitions that do not involve the spin flip of the tunneling electron are mediated by correlation. However, since they involve the small components in the state expansion over spin configurations, their probability is weak. Furthermore, if the length of the chain is increased, the direct 1-2 transition probability decreases rapidly. This is clearly seen in Fig. 2, which shows the $1 \rightarrow 2$ transition rate induced by tunneling electrons as a function of the STM bias. At low bias (below $6 \mathrm{mV}$ ), only direct $1 \rightarrow 2$ transitions are possible, with a very small transition probability for long chains that very rapidly decreases as the chain length increases. Typically, the direct $1 \rightarrow 2$ excitation probability per electron is equal to $0.9 \times 10^{-7}\left(1.5 \times 10^{-11}\right)$ for $\mathrm{Fe}_{6}\left(\mathrm{Fe}_{8}\right)$.

Above $6 \mathrm{mV}$, the transition rate increases drastically due to indirect transitions: The system initially in state 1 is

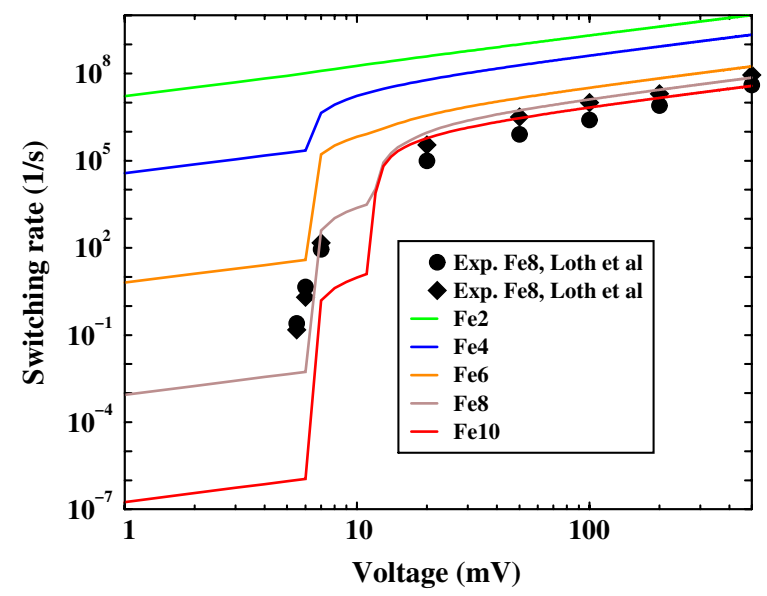

FIG. 2 (color online). Tunneling electron-induced switching rate between Néel states in $\mathrm{s}^{-1}$ as a function of the STM voltage in $\mathrm{mV}$. Lines: The present calculations using Eq. (5) for $\mathrm{Fe}$ chains of different lengths (see the inset for details); the STM current is set equal to $20 \mathrm{pA}$ at $2 \mathrm{mV}$ [12]. The higher rates correspond to the Fe dimer, and the rate decreases as the chain length increases. The experimental results [12] are plotted for transitions from low to high currents (dots) and from high to low currents (diamonds). excited by collision with the tunneling electron into a higher-lying state $i$, and state $i$ later decays into state 2 with a finite probability [Fig. 1(b)]. The decay process proceeds via electron-hole pair excitation of the substrate and it is the inverse of the excitation process discussed above [16]. The above strong-coupling approach successfully accounts for excited spin state lifetimes [16,28]. Hence, we can complete the full excitation or deexcitation dynamics by considering now the deexcitation probability $P_{i \rightarrow f}^{\text {Dec }}$ (vanishing temperature):

$$
P_{i \rightarrow f}^{\mathrm{Dec}}=\Delta E_{i j} \frac{P(i \rightarrow f)}{\sum_{l<i} P(i \rightarrow l) \Delta E_{i l}},
$$

where $P(i \rightarrow f)$ has been defined earlier and $\Delta E_{i j}=$ $E_{i}-E_{f}$ is the decay energy. Since there is no long-lived excited state in the system (except state 2), an excitation of state $i$, followed by successive decays according to (4), eventually results in the population of the two Néel states, $N_{i}(1)$ and $N_{i}(2)$. The $1 \rightarrow 2$ transition rate for a bias $V$ at $0 \mathrm{~K}$ is then given by

$$
\begin{aligned}
\operatorname{Rate}(1 \rightarrow 2)= & C\left[\left(\mathrm{eV}-\Delta E_{21}\right) P(1 \rightarrow 2)\right. \\
& \left.+\sum_{i>2}\left(\mathrm{eV}-\Delta E_{i 1}\right) P(1 \rightarrow i) N_{i}(2)\right] .
\end{aligned}
$$

The $C$ coefficient is the normalization constant for the total flux of electrons in a given experiment [29] which is bias independent for small bias. The first term in Eq. (5) corresponds to the direct transitions already discussed and the sum over $i$ to the indirect transitions.

Figure 2 shows the computed rates for $\mathrm{Fe}_{n}$ chains $(n=2-10)$ compared with the experimental results for $\mathrm{Fe}_{8}$ [12]. The theoretical transition rate decreases with the chain length, and from there one can conclude that direct transitions are practically impossible for very long chains whereas the indirect process should be accessible in a broad range of lengths. Our results on Fig. 2 compare very well with the experimental data for $\mathrm{Fe}_{8}$, also shown as dots and diamonds. Dots in Fig. 2 show the experimental transition rates from low current to high current, and diamonds form high to low currents. This corresponds to $1 \rightarrow 2$ transitions and $2 \rightarrow 1$, respectively. The experimental results in Fig. 2 were obtained with a polarized tip, whereas the theoretical results were obtained with a nonpolarized tip. Polarizing the tip influences the transition rates, due to the intermediate $S_{T}=5 / 2$ symmetry involved in the tunneling. Inelastic transitions are favored when the tip and the atom under the tip have opposite spin directions; the ratio between the theoretical switching rates is typically around 3 for a tip polarization equal to 0.3 , which explains the asymmetry between dots and diamonds in Fig. 2.

The theoretical switching rates show an abrupt change at $\sim 12 \mathrm{meV}$. This change is more clearly seen in $\mathrm{Fe}_{8}$ and 
$\mathrm{Fe}_{10}$ chains. By studying the spectra of excitations for these chains, we can clearly separate two distinct regions in the rate due to two sets of excitations different in nature. The first region, between 6 and $12 \mathrm{meV}$, corresponds to transitions where $M$ changes in one of the end atoms of the chain; these are very efficient in shorter chains such as $\mathrm{Fe}_{6}$. This type of excitation is a quantized spin wave of the finite chain [27]. The second region, beyond $12 \mathrm{meV}$, corresponds to transitions that mix several configurations with two opposite antiferromagnetic domains (domain-wall formation). As the chain length increases, domain-wallmediated transitions become more important than spinwave-mediated transitions, explaining the clear upturn beyond $12 \mathrm{meV}$ for $\mathrm{Fe}_{8}$ and $\mathrm{Fe}_{10}$. There is also a dependence of the transition rate on the position of the tip along the chain. Indeed, this position effect mixes with the polarization effect due to the alternating spin directions along the chain in the Néel state as well as with the effect of the position of the inhomogeneous term (2) along the chain. Besides the interplay with the polarization effects, end atoms are more efficient for moderate bias and central atoms for high voltages.

The transition path $1 \rightarrow i \rightarrow 2$ for $\mathrm{Fe}_{8}$ is analyzed in Fig. 3. The different contributions to the switching rate, Eq. (5), are presented as functions of the excitation energy: the primary excitation probability $P(1 \rightarrow i)$ (black circles), the branching ratio toward state $2\left[N_{i}(2)\right.$, red crosses], and the total indirect excitation probability $P(1 \rightarrow i) N_{i}(2)$ (green diamonds). Many states $i$ are excited by a tunneling electron, although many of them with a small probability that roughly exponentially decreases with the excitation energy [27]. The largest excitation probability corresponds to states $i$ where the spin of the

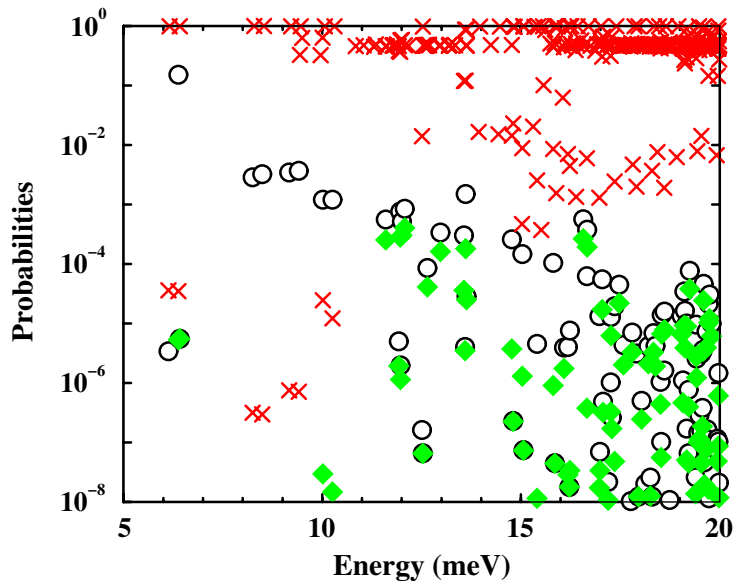

FIG. 3 (color online). Excitation probability from the Néel state 1 to level $i, P(1 \rightarrow i)$ (black circles); branching ratio toward state 2 once level $i$ is populated, $N_{i}(2)$ (red crosses); and the total indirect switching probability between Néel states 1 and 2, $P(1 \rightarrow i) N_{i}$ (2) (green diamonds). Many states $i$ are excited by an electron, with a probability roughly exponentially decreasing with the excitation energy, but the switching probability is strongly modulated by the deexcitation branching ratio. atom under the STM tip changes by $\Delta M \approx \pm 1$. All the other states are excited via correlation, i.e., the fact that states 1 and $i$ are not associated to a single configuration of local spins [27] but to a mixing of a large number of them from a configuration interaction point of view. As for the decay of the excited states, $i$, the lower-energy states decay preferentially toward Néel state 1 or 2, depending on which state they are configurationally closer. For high-lying states, correlation becomes stronger and the excited states decay roughly equally to the two Néel states. Domain walls, in particular, decay roughly equally to both Néel states. As a conclusion from Fig. 3, the global transition $1 \rightarrow i \rightarrow 2$ requires that both excitation and deexcitation are sizeable, i.e., requires balancing the $1 \rightarrow i$ and $i \rightarrow 2$ probabilities.

The relative weight of the various switching processes varies with the chain length, and this can be rationalized, considering that the distance between the two Néel states in terms of spin configuration changes is increasing with chain length. For the direct quasiresonant process at small bias, one needs a strong configuration mixing between Néel states, and this quickly decreases with chain length. The process around $6-8 \mathrm{meV}$ is associated to $\Delta M \approx \pm 1$ transitions in the atom under the tip; the corresponding excited state is still very close to the initial Néel state, and therefore the decay to the other Néel state is difficult and rapidly decreases with the chain length increase. The process around 12-13 meV, associated to domain-wall formation, behaves differently. First, several states contribute and their number increases with chain length; second, their decay equally populates the two Néel states. So, even if their excitation probability from the initial Néel state is not very high, they succeed in dominating the indirect process for long chains.

In summary, our calculations show three different regimes in the tunneling electron-induced switching of the AFM chains in Ref. [12]. The low-bias region corresponds to quasiresonant direct transitions between the two Néel states. The intermediate-bias region is characterized by the threshold of chain excitations. Beyond this threshold, tunneling electrons induce an indirect process mediated by spin-wave excitations. A second threshold defines the high-bias region where domain-wall excitations dominate the switching process. Correlation, i.e., mixing of spin configurations in the chain, is the driving force of the three Néel switching processes. However, correlation acts differently in the three, resulting in different dependences on the chain length of the three process and leading to the dominance of the process involving domain-wall formation for long chains.

The mechanisms described in this Letter are very different from the more usual local spin-flip mechanisms at play in, e.g., magnon excitation in ferromagnetic chains. Instead, the present mechanisms should be very general and operational in many systems with strong correlations, 
such as frustrated systems. As an example, the three processes unveiled in the present work for the tunneling electron-induced switching should also be active in the thermally activated switching process revealed by the experiment [12] (substrate electrons colliding on the chain can induce switching similarly to tunneling electrons); as a confirmation, one can stress that the activation energies found experimentally correspond to the excitation energies involved in the indirect process discussed here.

M. Y. and X.C. acknowledge financial support through Spain's MINECO under Contract No. TEC2009-06986.

[1] S.A. Wolf, D.D. Awschalom, R. A. Buhrman, J.M. Daughton, S. von Molnár, M.L. Roukes, A. Y. Chtchelkanova, and D. M. Treger, Science 294, 1488 (2001).

[2] L. Bogani and W. Wernsdorfer, Nat. Mater. 7, 179 (2008).

[3] B. Behin-Aein, D. Datta, S. Salahuddin, and S. Datta, Nat. Nanotechnol. 5, 266 (2010).

[4] S. S. P. Parkin, M. Hayashi, and L. Thomas, Science 320, 190 (2008).

[5] A. A. Khajetoorians, J. Wiebe, B. Chilian, and R. Wiesendanger, Science 332, 1062 (2011).

[6] A. R. Rocha, V. M. García-suárez, S. W. Bailey, C. J. Lambert, J. Ferrer, and S. Sanvito, Nat. Mater. 4, 335 (2005).

[7] M. Affronte, J. Mater. Chem. 19, 1731 (2009).

[8] M. Mannini et al., Nat. Mater. 8, 194 (2009).

[9] F. Troiani and M. Affronte, Chem. Soc. Rev. 40, 3119 (2011).

[10] F. Meier, J. Levy, and D. Loss, Phys. Rev. Lett. 90, 047901 (2003).

[11] A. Candini et al., Phys. Rev. Lett. 104, 037203 (2010).

[12] S. Loth, S. Baumann, C.P. Lutz, D. M. Eigler, and A. J. Heinrich, Science 335, 196 (2012).
[13] F. Delgado and J. Fernández-Rossier, Phys. Rev. Lett. 108, 196602 (2012).

[14] S. Loth, K. von Bergmann, M. Ternes, A. F. Otte, C. P. Lutz, and A. J. Heinrich, Nat. Phys. 6, 340 (2010).

[15] F. Delgado, J. J. Palacios, and J. Fernández-Rossier, Phys. Rev. Lett. 104, 026601 (2010).

[16] F. D. Novaes, N. Lorente, and J.-P. Gauyacq, Phys. Rev. B 82, 155401 (2010).

[17] J.-P. Gauyacq, N. Lorente, and F. D. Novaes, Prog. Surf. Sci. 87, 63 (2012).

[18] C.F. Hirjibehedin, C.-Y. Lin, A.F. Otte, M. Ternes, C. P. Lutz, B. A. Jones, and A. J. Heinrich, Science 317, 1199 (2007).

[19] N. Lorente and J.-P. Gauyacq, Phys. Rev. Lett. 103, 176601 (2009).

[20] C. F. Hirjibehedin, C. P. Lutz, and A. J. Heinrich, Science 312, 1021 (2006).

[21] A. N. Rudenko, V. V. Mazurenko, V.I. Anisimov, and A. I. Lichtenstein, Phys. Rev. B 79, 144418 (2009).

[22] S. Loth and A. Heinrich (private communication).

[23] Note that the $J$ and $D$ parameters are very close to the Ising representation in Ref. [12] as well as to the fitting of $D$ for a monomer.

[24] V. Hernández, J. E. Román, and V. Vidal, ACM Trans. Math. Softw. 31, 351 (2005).

[25] J.-P. Gauyacq, F. D. Novaes, and N. Lorente, Phys. Rev. B 81, 165423 (2010).

[26] K. Yosida, Theory of Magnetism (Springer, Berlin, 1991), p. 125.

[27] J.-P. Gauyacq and N. Lorente, Phys. Rev. B 83, 035418 (2011).

[28] J.-P. Gauyacq and N. Lorente, Phys. Rev. B 85, 115420 (2012).

[29] In the experiment of Ref. [12], the normalization constant is $C=6.25 \times 10^{7} \mathrm{~s}^{-1} \mathrm{meV}^{-1}$, corresponding to a current of $20 \mathrm{pA}$ at $2 \mathrm{mV}$. 